\title{
Introduction to self-organization in chemical and electrochemical systems
}

\author{
Marek Orlik ${ }^{1}$ \\ Received: 13 August 2015 / Accepted: 17 August 2015 / Published online: 2 September 2015 \\ (C) The Author(s) 2015. This article is published with open access at Springerlink.com
}

The world around us, and including us, is a huge dynamical system composed of dynamical subsystems in which, in course of irreversible processes, spontaneous organization of matter into patterns of different complexity, or simplySHAPES, takes place. Since we believe that the second law of thermodynamics cannot be violated, the entropy decrease assisting the formation of every pattern must be overcompensated by the entropy increase in simultaneously occurring dissipative processes. Since however not every irreversible process is a source of dynamic self-organization, in addition to this general thermodynamic condition, also, some additional strict kinetic criteria must be fulfilled: namely — the nonlinearity of the system's dynamics and the presence of appropriately operating feedback loops in the kinetic mechanism. Finally, the control parameters, like, e.g., rate constants, initial concentrations of reactants, electrode potential or electric current, or temperature have to be tuned up to the values that throw the system out of its trivial stable steady-state towards self-organized instabilities, like the oscillations of the entire system's state or spatial/spatiotemporal patterns. The concept of stability is thus crucial: the "exotic" selforganized dynamics is a consequence of a loss of stability of the "trivial" steady-state. The pulsations of our heart, the conduction of electric impulse along the axon, the oscillatory activity of our brain, the visually attractive patterns on the skins of animals, like zebras or snakes belong to the rich set of natural phenomena, the understanding of which is possible in terms of an interdisciplinary science, termed nonlinear dynamics. You might think that such impressive and crucial,

Marek Orlik

morlik@chem.uw.edu.pl

1 The University of Warsaw, Warsaw, Poland for functioning of living matter, phenomena should attract the attention of many researchers, as it was the case, among others, for the Nobel Prize winners: I. Prigogine, M. Eigen, and more recently G. Ertl. However, this is evidently not the case for the majority of chemical community and the main reason for that lack of interest seems to be an insufficient education. Principles of nonlinear dynamics are in fact being taught only at those few universities, where people are working who have a serious interest in this topic and who share their knowledge and enthusiasm with the students. In consequence, the vast majority of chemists of all generations, who received only traditional education in chemistry, even if they heard something about oscillations or related phenomena, are not familiar at all with their fascinating scientific background. Moreover, a conservative way of education favors perception of nonlinear dynamics as a rather difficult and exotic discipline, for which ordinary chemists are not prepared. This is of course not true since the education in this area, as in all other disciplines, is only a question of an appropriate effort and motivation. In consequence of all these reservations of the chemical community, limited interest in chemical nonlinear dynamics manifests itself also in the area of interfacial electrochemistry, for which it is even easier than in homogeneous chemistry to drive the systems smoothly out from equilibrium, by simply varying the electrode potential or imposed current.

Since most (electro)chemists are thus not familiar with nonlinear dynamics, it is useful to introduce here some of the basic concepts [1-9], in order to facilitate the reading of the contributions to this topical issue. If similar dynamic phenomena occur in different systems, they must have common mechanistic basis. From the physical point of view, the essential reason for the occurrence of the oscillatory behavior is the alternate domination of the positive and negative feedback loops in the system's dynamics. From the mathematical point of view, the transition from the non-oscillatory, steady-state to 
the oscillations is a manifestation of the respective bifurcation, being in many cases the one which is termed the Hopf (or Hopf-Andronov) bifurcation. In an appropriately defined phase space, such a bifurcation is graphically represented as a transition from the single attractive point (point attractor) to the cyclic attractive trajectory (cyclic attractor, limiting cycle) surrounding now unstable, thus repelling, single steady-state which lost the stability due to this bifurcation. The system's dynamics can be also more complicated, producing more complex, but still periodic oscillations (cf., e.g., mixed-mode, i.e., composed of small-amplitude and large-amplitude oscillations). Upon tuning the control parameters, these oscillations can become effectively aperiodic, so practically chaotic, but in some cases, it is possible to detect a complex order in this regime, described then as the "deterministic chaos." Its original discovery in numerical calculations, made by W. Lorenz in 1963 [10], led to surprising conclusion that only three ordinary differential equations, describing the system's dynamics, are sufficient to exhibit such complex behavior, the phase portraits of which are termed strange attractors. Besides the oscillations, another dynamic regime that can occur, and also only under non-equilibrium conditions, is multistability, meaning coexistence of more than one branch of stable steady-states for certain range of control parameters. In the simplest case of bistability, the possible steady-states plotted as a function of the control parameter form a single fold, the upper and the lower branches of which contain the available stable steady-states, while the middle section of the fold is composed of unstable, i.e., directly not observable steady-states. An important feature of such folded characteristics is the hysteresis in the system's response upon cyclic variation of the control parameter, since in the bistable region the system attains, for exactly the same value of the control parameter, either the upper or the lower branch of the stable states, depending on the starting value of the smoothly varied control parameter ("memory effect"). The transition between the "trivial" monostable and bistable regime is termed the saddle-node bifurcation. Finally, one should identify the phenomenon of excitability, known as characteristics of neurons upon external perturbation. The key feature of the excitable systems is the existence of a certain threshold in the magnitude of external perturbation, below which the response is relatively small and proportional to the perturbing signal, while above which an abrupt strong excitation takes place, with the amplitude being no longer dependent on the magnitude of this perturbation. Such characteristics were found also for both chemical and electrochemical dynamic systems and can be explained in terms of the models involving at least two variables significantly differing in the time scale of their dynamics. To conclude, non-equilibrium dynamical systems, depending on control parameters, can exhibit oscillations, multistability, and excitability, or only some of these phenomena.

In homogeneous chemistry, we look for the kinetic mechanisms that satisfy the conditions for occurrence of those exotic dynamic regimes. Interfacial electrochemical systems have of course their specific features which contribute to the source of instabilities, also of the entire electric circuit [3-9]. One of them can be the non-monotonic dependence between the current and the electrode potential, caused by, e.g., passivation of the electrode surface or an adsorption of an inhibitor in certain potential range. The resulting negative $\mathrm{d} E / \mathrm{d} I$ slope, for the current $I$ controlled by the rate of the charge-transfer step, formally means a negative differential resistance (NDR) which existence has sound consequences for the system's dynamics. Let us consider a most common case of the $\mathrm{N}$-shaped region of the NDR (N-NDR). In the case of total compensation of the ohmic drops $\left(I R_{\mathrm{S}}\right)$, with $R_{\mathrm{S}}$ meaning the serial ohmic resistance in the circuit, the smooth, e.g., linear change of the electrode potential will cause tracking, point by point, of the entire steady-state $I-E$ dependence, i.e., under such conditions, the entire $I-E$ plot appears to be composed of directly observable, stable steady-states. On the contrary, the control of the polarizing current will result in discontinuous jumps of the electrode potential at the extrema of the $I-E$ dependence, showing that the branch of a negative slope (NDR) is now not accessible in this variant of the experiment, or - in other words - the steadystates forming the NDR branch are unstable under galvanostatic conditions. Moreover, cyclic variations of the current will reveal hysteresis in the potential response to the actual current-in this way, electrochemical bistability under galvanostatic conditions will manifest itself. Analogous, but in details, different, dynamic characteristics are observed if sufficiently high serial ohmic resistance $R_{\mathrm{S}}$ is now placed in the circuit. Then, the N-letter $I-E$ shape, visualized as the dependence of $I$ on the voltage drop $U=E+I R_{\mathrm{S}}$, deforms in such a way that the NDR branch becomes destabilized and directly inaccessible under potentiostatic (meaning now constant $U$ ) conditions, i.e., there exists a certain range of external voltage $U$, within which the steady-state current can attain either a low, or a high value, compared to the middle range of the unstable steadystate currents. Let us note also that the N-NDR characteristics introduces a positive feedback in the system's dynamics, as the fluctuation of the electrode potential is then self-amplified until the region of positive differential resistance is attained. Now, if there is an additional source of slow negative feedback, coming, e.g., from a relatively slow diffusion transport of the reacting particles from the system's bulk to the electrode surface, the steady-states may undergo continuous destabilization and sustained oscillatory variations of the current can then emerge, showing in such a case their strict correlation with bistability. Under appropriate conditions and for more sophisticated system's characteristics, involving the so-called hidden $\mathrm{N}$-shaped negative resistance (HN-NDR) [5], also oscillations of the electrode potential can occur upon polarization of the electrode with constant current.

Now, let us consider inhomogeneous systems in which concentration or potential gradients exist and hence induce 
transport phenomena, like diffusion or migration. One of the most surprising conclusions drawn from both theoretical models and experimental practice is that the coupling of nonlinear chemical dynamics with transport phenomena can generate non-equilibrium spatiotemporal and even steady-state spatial patterns, like the ones predicted by A. Turing in his seminal paper [11] on chemical morphogenesis in reactiondiffusion systems. Diffusion is then unable to homogenize the system but is forced by autocatalytic chemical reaction to participate in maintaining the system's inhomogeneity. An analogous role can be played by migration of charged particles in electrochemical systems, when Turing-like patterns, reported for the first time by K. Krischer, and G. Ertl and coworkers $[12,13]$, emerged for electrode processes with the S-shaped region of negative differential resistance. In bistable/excitable systems, the interplay of transport and (electro)chemical kinetics leads, e.g., to the concentric or spiral waves of concentration/electrode potential, moving through the reactor space or along the electrode surface. Today, we understand that if the electrode process is characterized with (explicit or hidden) negative resistance, its coupling with migration/diffusion can lead to spatiotemporal patterns on the electrode surfaces.

Finally, the convective motion of the fluid also can reveal self-organization, as the famous hexagonal cells reported by H. Bénard in 1901 (!) [14] for thermal convection proved. In electrochemistry, convection can be generated by induced density gradients in the electrolyte, by electrohydrodynamic forces and also by the gradients of interfacial tension, in the latter case leading to the so-called Marangoni-type instabilities [9]. In one of the best known and visually attractive examples, driven by cyclic repetition of redox processes occurring at the surface mercury drop, periodic changes of interfacial tension cause rhythmic motion of the "beating mercury heart." Although the basic phenomenon, according to D. Avnir [15], was discovered by G. Lippmann already in 1873 [16], it is still possible to detect exotic dynamics in this only apparently simple dynamical system.

What is thus the present state of research in the area of chemical/electrochemical self-organized dynamical systems? There is obviously still some space for the investigation of single dynamical systems, if the detailed mechanisms underlying the onset of dynamic instabilities are not yet fully recognized. There is evidently a growing interest in spatially extended systems, which in electrochemistry mean either studies of pattern formation on the initially homogeneous single electrode surface, or investigations of wave propagation in various configurations of electrodes of different geometries. The dynamic cooperation of coupled systems, each of them being individually able to exhibit oscillatory or multistable behavior, is another promising direction, the more so because coupled dynamical systems resemble better the functioning of living matter than any single system. Recent years brought also attention to the studies of control of chaos and of unstable steady-states in electrochemical systems by applying specially designed, feedback-based, strategies of imposing perturbations [9]. Electrochemical dynamical systems, subject to periodic or chaotic external perturbation, can also exhibit various types of resonance, like the stochastic or coherence resonance. One of the most recent ideas in electrochemical nonlinear systems is searching for the so-called chimera states, i.e., dynamical regimes in which an array of identical oscillators splits into two domains: one coherent and phaselocked, and the other incoherent and desynchronized, according to original report of S.I. Shima and Y. Kuramoto from the year 2002 [17].

The nine papers composing this topical issue reflect the current state of research in the nonlinear electrochemical dynamics. Relevant experimental and theoretical studies refer to the processes of electrochemical passive/active transition in anodic dissolution of metals, electroreduction, and electrooxidation processes of small ions and molecules at solid electrodes, as well as to Marangoni-type instabilities at the mercury/solution interface. It is particularly instructive to note how apparently well-known and widely studied (at least from the point of view of classical electrochemistry) electrode processes can be used to design systems that exhibit various kinds of dynamic self-organization.

In conclusion, the guest editor and all the authors of the contributed works are very grateful to the Editor, Prof. Dr. F. Scholz for the idea of preparing this unique topical issue that, hopefully, will stimulate more researchers to undertake studies in this important, interdisciplinary, but still underestimated, due to insufficient education in nonlinear dynamics, branch of physical chemistry.

Marek Orlik

Guest Editor

Open Access This article is distributed under the terms of the Creative Commons Attribution 4.0 International License (http://creativecommons.org/licenses/by/4.0/), which permits unrestricted use, distribution, and reproduction in any medium, provided you give appropriate credit to the original author(s) and the source, provide a link to the Creative Commons license, and indicate if changes were made.

\section{References}

1. Nicolis G, Prigogine I (1977) Self-organization in nonequilibrium systems. From dissipative structures to order through fluctuations. Wiley-Blackwell

2. Strogatz SH (2015) Non-linear dynamics and chaos, 2nd Ed., Westview Press

3. Wójtowicz J (1973) Oscillatory behavior in electrochemical systems. In: O'M Bockris J, Conway BE, White RE (eds) Modern aspects of electrochemistry, vol 8. Plenum, New York

4. Hudson JL, Tsotsis TT (1994) Electrochemical reaction dynamics: a review. Chem Eng Sci 49(10):1493-1572 
5. Koper MTM (1996) Oscillations and complex dynamical bifurcations in electrochemical systems. In: Prigogine I, Rice SA (eds.) Adv Chem Phys XCII:161-298, Wiley

6. Krischer K (1999) Principles of temporal and spatial pattern formation in electrochemical systems. In: Conway BE, Bockris JO'M, White RE (eds) Modern aspects of electrochemistry, vol 32. Kluwer, New York

7. Krischer K (2001) Nonlinear dynamics in electrochemical systems. In: Alkire RC, Kolb DM (eds) Advances in electrochemical science and engineering, vol 8. Wiley-VCH, Weinheim

8. Orlik M (2012) Self-organization in electrochemical systems I. General principles of self-organization. Temporal instabilities. In: Scholz F (ed) Monographs in electrochemistry. Springer, Berlin

9. Orlik M (2012) Self-organization in electrochemical systems II. Spatiotemporal patterns and control of chaos. In: Scholz F (ed) Monographs in electrochemistry. Springer, Berlin
10. Lorenz EN (1963) Deterministic nonperiodic flow. J Atmos Sci 20: 130-141

11. Turing AM (1952) The chemical basis for morphogenesis. Philos Trans Soc Lond Ser B 327:37-72

12. Mazouz N, Krischer K (2001) A theoretical study on Turing patterns in electrochemical systems. J Phys Chem B 104:6081-6090

13. Li YJ, Oslonovitch J, Mazouz N, Plenge F, Krischer K, Ertl G (2001) Turing-type patterns on electrode surfaces. Science 291:2395-2398

14. Bénard H (1901) Les tourbillons cellulaires dans une nappe liquide transportant de la chaleur par convection en régime permanent. Ann Chem Phys 23:62-144

15. Avnir D (1989) Chemically induced pulsations of interfaces: the mercury beating heart. J Chem Educ 66:211-212

16. Lippmann G (1873) Ann Phys Ser 2 149:546

17. Shima SI, Kuramoto Y (2002) Coexistence of coherence and incoherence in nonlocally coupled phase oscillators. Nonlin Phenom Complex Syst 5:380-385 